\title{
Implementing an acute pain unit focused on continuous regional analgesia at home.
}

\section{Implementando una unidad de dolor agudo enfocada en analgesia regional continua domiciliaria. \\ Https://doi.org/10.25237/carsach2020.09}

Dr. Pablo Miranda H. ${ }^{1}$ Dra. Andrea Araneda V. ${ }^{1}$ Dra. Francisca Elgueta L. ${ }^{1}$ Dr. Fernando Rueda B. ${ }^{1}$ Dr. Fernando Altermatt C. ${ }^{1}$

${ }^{1}$ División de Anestesiología. Escuela de Medicina. Pontificia Universidad Católica de Chile.

Autor Corresponsal:

Dr. Pablo Miranda H.

División de Anestesiología. Escuela de Medicina. Pontificia Universidad Católica de Chile.

Dirección: Marcoleta 367, Santiago, 8330024, Chile

Teléfono: 56223543270

e-mail: pablofmh@gmail.com

ORCID ID: https://orcid.org/0000-0003-2200-0186

Palabras claves: analgesia post operatoria, bloqueo continuo de nervio periférico continuo, analgesia regional continua domiciliaria, cirugía ambulatoria.

Key words: post-operative analgesia, continuous peripheral nerve blocks, continuous regional analgesia at home, ambulatory surgery.

\section{Puntos clave}

- Los bloqueos de nervio periférico continuos proveen una excelente analgesia de larga duración en un sitio específico.

- Comparados con pacientes que reciben analgesia sistémica con opioides, los pacientes con analgesia regional continua presentan menos dolor y menos efectos adversos a opioides.

- No todos los pacientes son buenos candidatos para la utilización de un sistema de analgesia regional continua domiciliaria. Se debe considerar las características del paciente, magnitud de dolor postoperatorio y la necesidad de terapia física.

- La adecuada selección de pacientes, educación previo al alta y el seguimiento son la clave del éxito de un sistema de analgesia regional continua domiciliaria.

- La coordinación de un sistema de analgesia regional continua domiciliaria debe estar a cargo de una unidad de dolor, la cual debe tener capacidad de respuesta 24/7 ante eventuales complicaciones.

- La unidad de dolor agudo debe estar constantemente evaluando sus resultados. 


\title{
Resumen
}

Dentro de los problemas más frecuentes y desafiantes de la cirugía ambulatoria se encuentra el adecuado manejo del dolor, en ese sentido los bloqueos de nervio periféricos continuos proveen una excelente analgesia, de larga duración con escasos efectos adversos. Incluso los pacientes se pueden enviar a sus domicilios con un dispositivo de infusión de anestésicos locales a través de los catéteres, sistema que de denomina analgesia regional continua domiciliaria. Para el buen funcionamiento de este sistema analgésico, los pacientes deben ser adecuadamente seleccionados previamente, educados antes del alta y realizarles seguimiento, logística que debe estar coordinada por una unidad de dolor.

La puesta en marcha de una unidad de dolor que esté a cargo de pacientes con analgesia regional continua domiciliaria presenta una serie de desafíos que presentamos en la siguiente revisión.

\begin{abstract}
Pain management is one of the most frequent and challenging problems in ambulatory surgery. Continuous peripheral nerve blocks provide an excellent long-lasting analgesia with few side effects. Patients can be sent home with a system known as continuous domiciliary regional analgesia, which consists in a device that infuses local anesthetics through catheters. In order for this analgesic system to work properly, patients must be adequately selected. They must be educated before medical discharge and they must be monitored throughout the whole process. An Acute Pain Unit must coordinate all the logistics involved.

The implementation of an Acute Pain Unit in charge of patients with continuous domiciliary regional analgesia presents a series of challenges that we detail in the following review.
\end{abstract}

\section{Introducción}

Uno de los grandes desafíos de la cirugía ambulatoria es el adecuado manejo del dolor, siendo éste, causa frecuente de consulta en el servicio de urgencia o de readmisiones no programadas (1). La literatura es clara en demostrar la superioridad en cuanto al manejo del dolor de la analgesia regional continua (ARC) en relación a la analgesia sistémica (2-5). La ARC además, está asociada a menores niveles de sedación, náuseas y prurito(6) lo que la convierte en una excelente alternativa para poder dar de alta precozmente a los pacientes a sus domicilios o realizar cirugía ambulatoria. Este aspecto cobra especial relevancia en los tiempos de COVID-19, en relación al creciente interés de que los pacientes permanezcan el menor tiempo posible en un recinto hospitalario (7).

La Analgesia Regional Continua Domiciliaria (ARCD), consiste en enviar pacientes a su domicilio con un sistema de infusión de anestésico local través de un catéter insertado de forma percutánea en la cercanía de un nervio o plexo, con el objetivo de prolongar la analgesia por el tiempo que dure la infusión. Este sistema analgésico se ha utilizado de forma exitosa y segura, desde hace más de 20 años (8), con experiencias nacionales e internacionales tanto en pacientes pediátricos (9-10) como adultos $(2,11)$. Sin embargo la implementación plantea una serie de desafíos, más allá de la correcta inserción del catéter, para su adecuado funcionamiento; elementos que debe manejar y coordinar una unidad de dolor. Entre los aspectos a considerar, son fundamentales, la correcta selección del paciente, educación previo al alta y su seguimiento. Además, la unidad debe estar disponible las 24 horas del día y tener la capacidad de respuesta en caso que se presenten complicaciones.

\section{Unidad de Dolor Agudo enfocada en Analgesia Regional Continua Domiciliaria}

Ya en los años 60 se comienza a hablar de unidades organizadas cuyo objetivo principal es el manejo del dolor postoperatorio, las cuales debían tener un enfoque en el trabajo de equipo (12), sin embargo no es hasta sino mediados de los años 80 en donde se crean y comienzan a funcionar en Estados Unidos (13-14) y Europa (15) las primeras unidades de dolor agudo (UDA). Estas unidades tienen inicialmente a su cargo pacientes hospitalizados.

Hoy en día se entiende una UDA como un grupo de profesionales de diferentes áreas y especialidades (anestesiólogos, neurólogos, enfermeras, kinesiólogos, psicólogos, etc.) cuya finalidad es ayudar a los pacientes en el alivio del dolor para que puedan ser dados de alta lo más precozmente posible y reintegrarse a las actividades de la vida diaria. 
La UDA va a tener a su cargo pacientes hospitalizados y en caso que el centro cuente con ARCD esta unidad es la que debe coordinar los procesos de tal forma que el sistema funcione de forma segura para los pacientes.

\section{Aspectos a considerar para una adecuada Analgesia Regional Continua Domiciliaria}

\section{Selección de Pacientes}

La adecuada selección de pacientes que sean candidatos para ser enviados a su domicilio con una infusión perineural continua de anestésicos locales, es probablemente la clave para el éxito del sistema. Actualmente no disponemos de guías clínicas respecto a criterios uniformes en relación a la selección de pacientes, por lo que ésta finalmente se debe realizar caso a caso tomando en cuenta las diversas realidades de los diferentes centros y la experiencia del equipo. En el caso de los pacientes pediátricos se debe incorporar a los padres en la toma de decisiones.

Hay elementos que contraindican la anestesia regional y por consiguiente la ARCD como son: Rechazo del paciente o de los padres en el caso de pacientes pediátricos, infección del sitio de punción, alergia a las drogas administradas y alteración de la coagulación (16). Con el fin de evitar el riesgo de intoxicación sistémica por anestésicos locales (ISAL) los pacientes con falla renal o hepática deben ser excluidos como candidatos a utilizar una infusión continua de anestésicos locales en su domicilio (17). Para infusiones que potencialmente afectan al nervio frénico y provocan parálisis de del diafragma ipsilateral (p. Ej. Bloqueo interescalénico) se debe tener especial precaución en pacientes que tengan alguna patología pulmonar o cardíaca y en pacientes obesos $(4,18,19)$.

Para poder dar de alta a los pacientes se les debe entregar por escrito las instrucciones, por lo tanto es fundamental para poder seleccionar un paciente que este o sus cuidadores sean capaces de comprender dichas instrucciones, deben ser capaces de entender la escala de dolor utilizada en el centro, de observar la bomba, el catéter y la piel desde donde sale el catéter. Recordemos que estamos delegando en el paciente o sus cuidadores algunos aspectos del cuidado que clásicamente se han hecho en un centro hospitalario a cargo de profesionales capacitados. Es altamente recomendable que los pacientes firmen un consentimiento informado que describa claramente los potenciales riesgo de la técnica.

La comunicación del paciente con la UDA es un elemento central. Por un lado los profesionales de la UDA deben tener contacto al menos 1 vez al día mientras el paciente esté con el dispositivo de infusión y el paciente debe tener la forma de contactarse de manera efectiva con la UDA en caso de dudas o de presentar cualquier complicación. La UDA debe estar disponible las 24 horas del día y los 7 días de la semana con personal capacitado para responder solicitudes por parte de los pacientes. Esto implica que se debe contar con un sistema de registro de la información que debe estar disponible en todo momento.

Finalmente, otro elemento importante a la hora de seleccionar los pacientes es la accesibilidad a un centro se salud. Esto ya que se puede presentar alguna complicación y el paciente necesite volver. En este punto no hay evidencia clara ni un consenso absoluto, sin embargo, parece razonable estar a una distancia que permita regresar en menos de 1 hora (9) (Tabla 1).

\section{Educación pre alta}

Previo a la instalación del dispositivo de infusión de anestésicos locales al catéter de nervio periférico, se recomienda aspirar el catéter para descartar una posición intravascular e inyectar un volumen mínimo para asegurarse que no esté ocluido. Al momento del alta además de quedar con el dispositivo de infusión de anestésico local, de no presentar contraindicaciones, se les deben prescribir otros medicamentos vía oral por horario (AINES, Paracetamol) y de rescate. Se debe educar en la correcta utilización de la analgesia de rescate y cuando pedir ayuda contactando a la UDA (20).

Previo al alta los pacientes o cuidadores deben recibir educación en relación a los cuidados que deben tener con la bomba, se recomienda dejarla en un lugar fijo, que no se desplace con los movimientos (ej. Bolso colgante alrededor del cuello) de tal forma de evitar que se caiga y pueda retirar el catéter al traccionarlo (Foto 1). Se debe hacer especial hincapié en señalar que la extremidad va a estar con pérdida de sensibilidad, por lo que se deben evitar elementos que la puedan dañar tales como frío o calor.

A los pacientes se les debe educar en la utilización de la escala de dolor utilizada en el centro acorde a la edad, esta va a ser la forma de cuantificar el dolor mientras el paciente esté en su domicilio. Se les debe explicar una forma sencilla de evaluar el bloqueo sensitivo y motor lo cual se debe consignar dentro de los datos del seguimiento. 
Todas estas indicaciones, incluyendo el teléfono y correo electrónico de la UDA y posibles complicaciones por las cuales deban consultar deben quedar por escrito (21) (Foto 2). La bomba elastomérica o el dispositivo que se utilice en el centro, debe llevar el nombre del paciente, RUT, medicamento utilizado, dilución, fecha y hora de instalación. (Foto 3).

\section{Seguimiento y retiro de catéter}

La forma y frecuencia en que se debe contactar a los pacientes en el domicilio es un tema controversial, algunos centros reportan visitas domiciliarias dos veces al día por enfermeras (22), sin embargo, la mayoría de los protocolos contactan a los pacientes una vez al día de forma telefónica mientras la bomba elastomérica esté funcionando $(2,5)$. No disponemos de evidencia actual para señalar que un método sea superior sobre otro, sin embargo, lo importante es que, independiente de la forma y la frecuencia de contacto, se debe estar siempre atento a pesquisar las complicaciones.

$\mathrm{Al}$ momento de contactar a los pacientes se les debe preguntar por la magnitud del dolor utilizando una escala local, grado de bloqueo sensitivo y motor, utilización de analgesia de rescate, signos o síntomas de ISAL, caídas y/o alguna otra lesión. Se le debe pedir que observe el sitio de inserción del catéter y que no presente alteraciones y que observe la bomba de infusión para consignar que ésta se vaya vaciando (Foto 4). Todo lo que ocurre con estos pacientes en su domicilio debe ser debidamente documentado y registrado con el mismo estándar de un paciente hospitalizado (23).

En cuanto al retiro del catéter, existen varias formas de hacerlo: que el paciente acuda a un recinto hospitalario y que un profesional le retire el catéter (24) o que el retiro sea realizado en el domicilio ya sea por el paciente o su cuidador $(2,5,25)$. No disponemos de evidencia que demuestre la superioridad de una estrategia sobre la otra, sin embargo, la forma de retiro debe quedar claramente explicitada previo al alta y se debe consignar que el catéter salió por completo. Una forma sencilla de asegurarnos de aquello es observar la punta del catéter la cual tiene un color diferente.

\section{Complicaciones}

$\mathrm{Al}$ igual que todos los procedimientos médicos, la utilización de ARCD debe sopesar los beneficios con los potenciales riesgos, los cuales se le deben explicar de forma clara a los pacientes. Desafortunadamente hay mucha disparidad en las técnicas de inserción de los catéteres, equipamiento, fijación, flujo y concentración de las infusiones, localizaciones de los catéteres y experiencia del operador que hacen muy difícil generalizar las tasas de complicaciones para un bloqueo continuo en particular. Afortunadamente las complicaciones serias con daño permanente son muy infrecuentes y las complicaciones menores tienen una incidencia similar a las punciones únicas (26).

Dentro de las complicaciones más frecuentes está el retiro accidental del catéter que oscila entre un 1,8 a 18\% (27-30) y la falla secundaria que está descrita entre $1 \%$ y $50 \%(31,32)$. Otras complicaciones que podemos mencionar son el desplazamiento del catéter (31), la filtración de liquido a través del sitio de inserción (33), mal funcionamiento de la bomba (27) y desconexión accidental de ésta (5). Hay reportes anecdóticos de complicaciones más graves como son anudamiento del catéter (34), retención (35) y rotura del catéter (36).

En cuanto a las complicaciones infecciosas de la ARCD, se presenta en menos del $1 \%$ de los pacientes $(37,38)$ mientras que la inflamación del sitio de inserción puede ocurrir hasta en un 4\% (28) y la colonización bacteriana del catéter llegar hasta en un 57\% de los pacientes $(39,40)$. Dentro de los factores de riesgo descritos para la infección de un catéter de nervio periférico es que la duración sea mayor a 48 horas, ubicación femoral y axilar, ausencia de profilaxis antibiótica y diabetes $(38,41)$.

Como en todo bloqueo de nervio periférico, el daño neurológico también es una potencial complicación. Además de los factores asociados de la cirugía, al paciente y al bloqueo e inserción del catéter, otro elemento que se debe considerar como potencial causa de daño neurológico es la lesión por presión en una extremidad insensible, en especial cuando se usan yeso o férula (37). El daño permanente que dura más allá de 9 meses está reportado en hasta 4 a 7 por 10.000 pacientes con catéteres de nervio periférico $(42,43)$. El daño neurológico transitorio puede ocurrir hasta en un $3 \%$ de estos pacientes $(28,44)$. Es importante señalar que actualmente no existen estudios que comparen directamente si la incidencia de complicaciones neurológicas con catéteres de nervio periférico es distinta entre pacientes hospitalizados y ambulatorios.

Otra potencial complicación es el riesgo de caídas. Existe asociación entre bloqueos continuos del nervio femoral y caídas después de una artroplastía de cadera y rodilla (45), posiblemente causada por la debilidad del cuádriceps. Este tema es fundamental al momento de seleccionar y educar a los pacientes previo al alta, además de considerar utilizar 
intervenciones que puedan disminuir el riesgo de caídas como la utilización de bastones o inmovilizador en la rodilla durante la deambulación (46).

La ISAL es una complicación extremadamente poco frecuente, pero potencialmente muy grave considerando que los pacientes están lejanos a un centro asistencial. Los pacientes y sus cuidadores deben recibir y entender cabalmente las instrucciones respecto a síntomas precoces de intoxicación, y ser capaces de interrumpir la infusión a la espera de las instrucciones del médico (11).

En cuanto a las complicaciones hemorrágicas, hay reportes de casos de hematoma en las cercanías del catéter (47) en especial cuando se utiliza, de forma concomitante, heparina de bajo peso molecular como profilaxis $(48,49)$. La gran mayoría son autolimitados, sin embargo, se han descrito casos aislados que necesitan drenaje quirúrgico (47). Es por esto que es de vital importancia tener una buena comunicación con el equipo quirúrgico para coordinar este tipo de fármacos.

Además, esta descrita de forma anecdótica el vaciamiento del contenido de la bomba elastomérica en pocos minutos, por lo que hay que hacer especial hincapié en que los pacientes estén continuamente observando este dispositivo (29) (Tabla 2).

\section{Estrategia de Implementación}

La puesta en marcha de una UDA con la capacidad de atender pacientes con ARCD no es fácil, el cambio cultural de delegar en los pacientes, responsabilidades que históricamente han sido del equipo de salud es probablemente lo más difícil. Hasta el momento no contamos con guías de práctica clínica que señalen los elementos necesarios o requisitos que debe contar una unidad para poder hacerse cargo de pacientes con ARCD, por lo que mucha de la información está basada en los pacientes con analgesia regional continúa hospitalizados y en la experiencia de los distintos centros que a su vez cuentan con realidades muy diferentes. En nuestro centro tenemos una buena casuística y es una alternativa considerada como el estándar de cuidado en cirugías seleccionadas.

La ARCD tiene su nicho en ser una excelente alternativa analgésica en cirugías ambulatorias o con hospitalización abreviada en donde esperamos que el dolor postoperatorio sea moderado a severo. Por lo tanto, en primer lugar, nos debemos preguntar si en el centro donde se pretende implementar la UDA para hacerse cargo de pacientes ambulatorios, se realizan este tipo de cirugías.

Como hemos señalado, al estar los pacientes con una infusión continua de anestésicos locales, estos deben tener una forma expedita y rápida de comunicarse con la UDA las 24 horas del día, los 7 días de la semana, lo que lo convierte en un requisito indispensable para implementar un programa de ARCD. En el caso que al paciente se le indique asistir al centro asistencial, este debe atender las 24 horas y, en caso que la cirugía se realice en un centro de cirugía ambulatoria que no trabaje las 24 horas, se debe tener algún convenio con un centro que pueda resolver esas situaciones.

Otro elemento importante es la generación de protocolos de atención, los cuales deben estar escritos para el conocimiento de todo el equipo. El concepto es que una UDA debe funcionar independiente de las personas y con un estándar que se debe establecer de antemano. Se deben generar instructivos que se le entregan a los pacientes previo al alta en donde quede claro la forma en que el paciente se puede contactar con la unidad de dolor.

Se debe contar con un sistema de registro que contenga toda la información aportada por el paciente desde su domicilio, con un estándar similar al de un paciente hospitalizado.

Nosotros y otros grupos sugieren comenzar de forma progresiva, $(20,50)$ de tal forma de poder ir perfeccionando el funcionamiento a medida que se acumula experiencia. Esto sirve como aprendizaje para todo el equipo que se vea involucrado en el manejo de los pacientes.

Finalmente, se debe hacer un análisis periódico de toda la información acumulada, ésto como un mecanismo elemental de control de la calidad, efectividad y eventos adversos presentados en el sistema de ARCD. Un análisis de este tipo es la única forma de saber en que estamos fallando y cómo podemos mejorar (Tabla 3).

\section{Referencias}

1. Chung F, Ritchie E, Su J. Postoperative pain in ambulatory surgery. Anesth Analg 1997; 85: 808-16.

2. Ilfeld BM, Morey TE, Wang RD, et al. Continuous popliteal sciatic nerve block for postoperative pain control at home: a randomized, double-blinded, placebocontrolled study. Anesthesiology 2002;97(4):959-65. 
3. Borgeat A, Tewes E, Biasca N, et al. Patient-controlled interscalene analgesia with ropivacaine after major shoulder surgery: PCIA vs PCA. Br J Anaesth 1998;81(4):603-5.

4. Borgeat A, Perschak H, Bird P, et al. Patient-controlled interscalene analgesia with ropivacaine $0.2 \%$ versus patient-controlled intravenous analgesia after major shoulder surgery: effects on diaphragmatic and respiratory function. Anesthesiology 2000;92(1):102-8.

5. Ilfeld BM, Morey TE, Wright TW, et al. Continuous interscalene brachial plexus block for postoperative pain control at home: a randomized, double-blinded, placebo-controlled study. Anesth Analg 2003;96(4):1089-95.

6. Richman JM, Liu SS, Courpas G, et al. Does continuous peripheral nerve block provide superior pain control to opioids? A meta-analysis. Anesth Analg 2006; 102(1):248-57.

7. Recomendaciones para la Reorganización y Ampliación Progresiva de los Servicios de Salud para la Respuesta a la Pandemia de COVID-19 (Marzo 2020). Organización Panamericada de la Salud.

8. Rawal N, Axelsson K, Hylander J, et al. Postoperative patient- controlled local anesthetic administration at home. Anesth Analg1998;86:86-9.

9. Ludot H, Berger J, Pichenot V, et al. Continuous Peripheral Nerve Block for Postoperative Pain Control at Home: A Prospective Feasibility Study in Children. Reg Anesth Pain Med 2008; 33 (1): 52-6.

10. Corvetto M, Rattalino M, Altermatt F. Bloqueos Continuos de Nervio Periférico Ambulatorios en Pediatría: dos casos clínicos. Rev Chil Anest 2010; 39: 93-8.

11. Altermatt F, Corvetto M, De la Cuadra JC. Bloqueos perineurales continuos ambulatorios para cirugía ortopédica: experiencia inicial en el uso de bombas elastoméricas en domicilio. Rev Chil Anest 2006; 35: $91-7$.

12. Avery-Jones F. Postoperative pain. In: Bailey H, ed. Pye’s surgical handicraft. Bristol, UK: Wright, 1961:197206.

13. Ready LB, Oden R, Chadwick HS, et al. Development of an anesthesiology-based postoperative pain management service. Anesthesiology 1988; 68:100-6.

14. Petrakis JK. Acute pain services in a community hospital. Clin J Pain 1989; 5(1):S34-41.

15. Maier C, Kibbel K, Mercker S, et al. Postoperative pain therapy at general nursing stations: an analysis of eight year's experience at an anesthesiological acute pain service. Anaesthesist 1994; 43:385-97.

16. Liu S, Salinas F. Continuous plexus and peripheral nerve blocks for postoperative analgesia. Anesth Analg 2003; 96 (1) :263-72.

17. Denson DD, Raj PP, Saldahna F, et al. Continuous perineural infusion of bupivacaine for prolonged analgesia: pharmacokinetic considerations. Int J Clin Pharmacol Ther Toxicol 1983; 21:591-7.

18. Renes SH, van Geffen GJ, Rettig HC, et al. Minimum effective volume of local anesthetic for shoulder analgesia by ultrasound-guided block at root C7 with assessment of pulmonary function. Reg Anesth Pain Med 2010; 35:529 34.

19. Sardesai AM, Chakrabarti AJ, Denny NM. Lower lobe collapse during continuous interscalene brachial plexus local anesthesia at home. Reg Anesth Pain Med 2004; 29:65 - 8.

20. De la Cuadra JC, Corvetto M. Recomendaciones en técnicas de bloqueos continuos de nervio periférico. Rev Chil Anest 2011; 40: 224-229.

21. Grant SA, Neilsen KC. Continuous peripheral nerve catheters for ambulatory anesthesia. Curr Anesthesiology Rep 2000; $304-7$.

22. Macaire P, Gaertner E, Capdevila X. Continuous post-operative regional analgesia at home. Minerva Anestesiol 2001;67:109-16.

23. Ilfeld B, Enneking F. Continuous Peripheral Nerve Blocks at Home: A Review. Anesth Analg 2005;100:1822-33.

24. Chelly JE, Greger J, Gebhard R. Ambulatory continuous perineural infusion: are we ready? Anesthesiology 2000;93:581-2. 
25. Klein SM, Greengrass RA, Gleason DH, et al. Major ambulatory surgery with continuous regional anesthesia and a disposable infusion pump. Anesthesiology 1999;91:563-5.

26. Borgeat A, Ekatodramis G, Kalberer F, et al. Acute and nonacute complications associated with interscalene block and shoulder surgery: a prospective study. Anesthesiology 2001; 95:875 - 880.

27. Capdevila X, Pirat P, Bringuier S. Continuous Peripheral Nerve Blocks in Hospital Wards after Orthopedic Surgery: A Multicenter Prospective Analysis of the Quality of Postoperative Analgesia and Complications in 1416 Patients. Anesthesiology 2005;103: 1035-45.

28. Gurnaney H, Kraemer W, Maxwell L, et al. Ambulatory Continuous Peripheral Nerve Blocks in Children and Adolescents: A Longitudinal 8-Year Single Center Study. Anesth Analg 2014;118:621-7.

29. Ganapathy S, Amendola A, Lichfield R, et al. Elastomeric pumps for ambulatory patient controlled regional analgesia. Can J Anesth 2000; 47 (9): 897-902.

30. Araneda A, Miranda P, Corvetto M, et al. Retrospective analysis of adverse events associated with continuous peripheral nerve blocks at home during 2013-2014. Reg Anesth Pain Med 2015; 40 (1) e103-e104.

31. Neuburger M, Breitbarth J, Reisig F, et al. Complications and adverse events in continuous peripheral regional anesthesia: results of investigations on 3,491 catheters. Anaesthesist 2006;55:33-40.

32. Ganapathy S, Wasserman RA, Watson JT, et al. Modified continuous femoral three-in-one block for postoperative pain after total knee arthroplasty. Anesth Analg 1999;89:1197-202.

33. Capdevila X, Dadure C, Bringuier S, et al. Effect of patient-controlled perineural analgesia on rehabilitation and pain after ambulatory orthopedic surgery: a multicenter randomized trial. Anesthesiology 2006;105:566-73.

34. Offerdahl MR, Lennon RL, Horlocker TT. Successful removal of a knotted fascia iliaca catheter: principles of patient positioning for peripheral nerve catheter extraction. Anesth Analg 2004;99:1550 -2.

35. Buckenmaier CC, Auton AA, Flournoy WS. Continuous peripheral nerve block catheter tip adhesion in a rat model. Acta Anaesthesiol Scand 2006;50:694 - 8.

36. Chin KJ, Chee V. Perforation of a Pajunk stimulating catheter after traction-induced damage. Reg Anesth Pain Med 2006;31: 389 -90.

37. Swenson JD, Bay N, Loose E, et al. Outpatient management of continuous peripheral nerve catheters placed using ultrasound guidance: an experience in 620 patients. Anesth Analg 2006;103(6):1436-43.

38. Capdevila X, Bringuier S, Borgeat A. Infectious risk of continuous peripheral nerve blocks. Anesthesiology 2009;110(1):182-8.

39. Compere V, Legrand JF, Guitard PG, et al. Bacterial colonization after tunneling in 402 perineural catheters: a prospective study. Anesth Analg 2009;108:1326 -30.

40. Cuvillon P, Ripart J, Lalourcey L, et al. The continuous femoral nerve block catheter for postoperative analgesia: bacterial colonization, infectious rate and adverse effects. Anesth Analg 2001;93:1045-9.

41. Bomberg H, Kubulus C, List F, et al. Diabetes: a risk factor for catheter-associated infections. Reg Anesth Pain Med 2015; 40:16-21.

42. Ilfeld BM. Continuous peripheral nerve blocks: a review of the published evidence. Anesth Analg 2011; 113:904 -925 .

43. Barrington MJ, Watts SA, Gledhill SR, et al. Preliminary results of the Australasian Regional Anaesthesia Collaboration: a prospective audit of more than 7000 peripheral nerve and plexus blocks for neurologic and other complications. Reg Anesth Pain Med 2009; 34:534 - 541.

44. Brull R, McCartney CJ, Chan VW, El Beheiry H. Neurological complications after regional anesthesia: contemporary estimates of risk. Anesth Analg 2007; 104:965 - 974.

45. Ilfeld BM, Duke KB, Donohue MC. The association between lower extremity continuous peripheral nerve blocks and patient falls after knee and hip arthroplasty. Anesth Analg 2010;111:1552- 4. 
46. Muraskin SI, Conrad B, Zheng N, et al. Falls associated with lower-extremity-nerve blocks: a pilot investigation of mechanisms. Reg Anesth Pain Med 2007;32: 67-72.

47. Wiegel M, Gottschaldt U, Hennebach R, et al. Complications and adverse effects associated with continuous peripheral nerve blocks in orthopedic patients. Anesth Analg 2007;104:1578 - 82.

48. Klein SM, D'Ercole F, Greengrass RA, et al. Enoxaparin associated with psoas hematoma and lumbar plexopathy after lumbar plexus block. Anesthesiology 1997;87:1576-9

49. Bickler P, Brandes J, Lee M, et al. Bleeding complications from femoral and sciatic nerve catheters in patients receiving low molecular weight heparin. Anesth Analg 2006;103:1036-7.

50. Collado F, Aragón MC, Pérez AC, et al. Organización de una Unidad de Dolor Agudo en un Hospital Regional. Rev. Soc. Esp. Dolor 2008; 15 (1): 28-40.

\section{Tabla 1}

Elementos importantes a considerar en la selección de pacientes candidatos a analgesia regional continua domiciliaria.

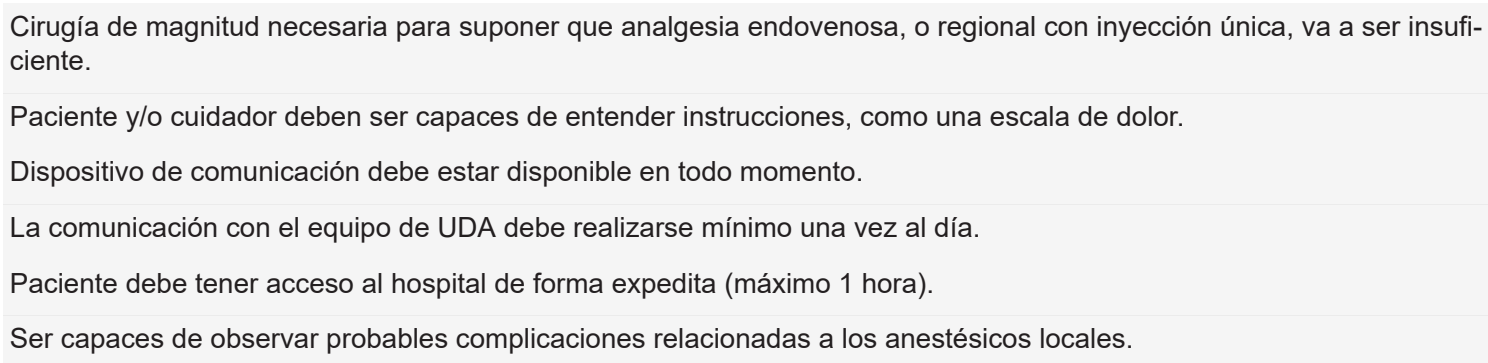

\section{Tabla 2}

\section{Complicaciones de la analgesia regional continua domiciliaria.}

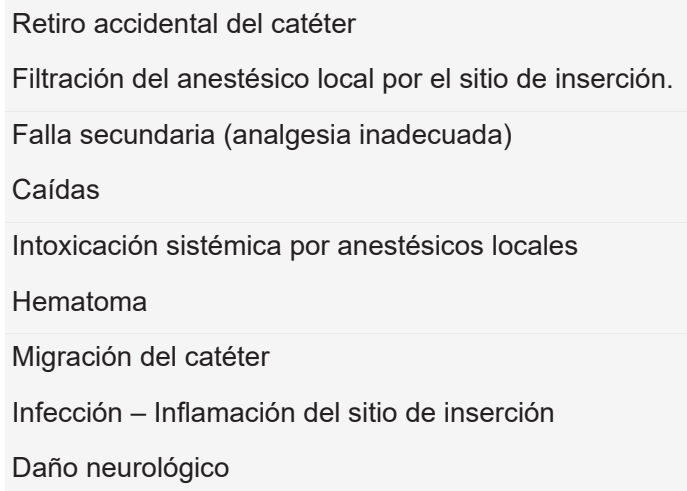


Tabla 3

Implementación de una unidad de dolor agudo enfocada en analgesia regional continua domiciliaria.

Ver las necesidades del centro

Generar protocolos de atención

Generar instructivos

Sistema de registro

Socializarlo con equipo de salud

Educación personal de trabajo

Comienzo progresivo - plan piloto

Medir resultados y solucionar problemas plan piloto

Implementación definitiva. 


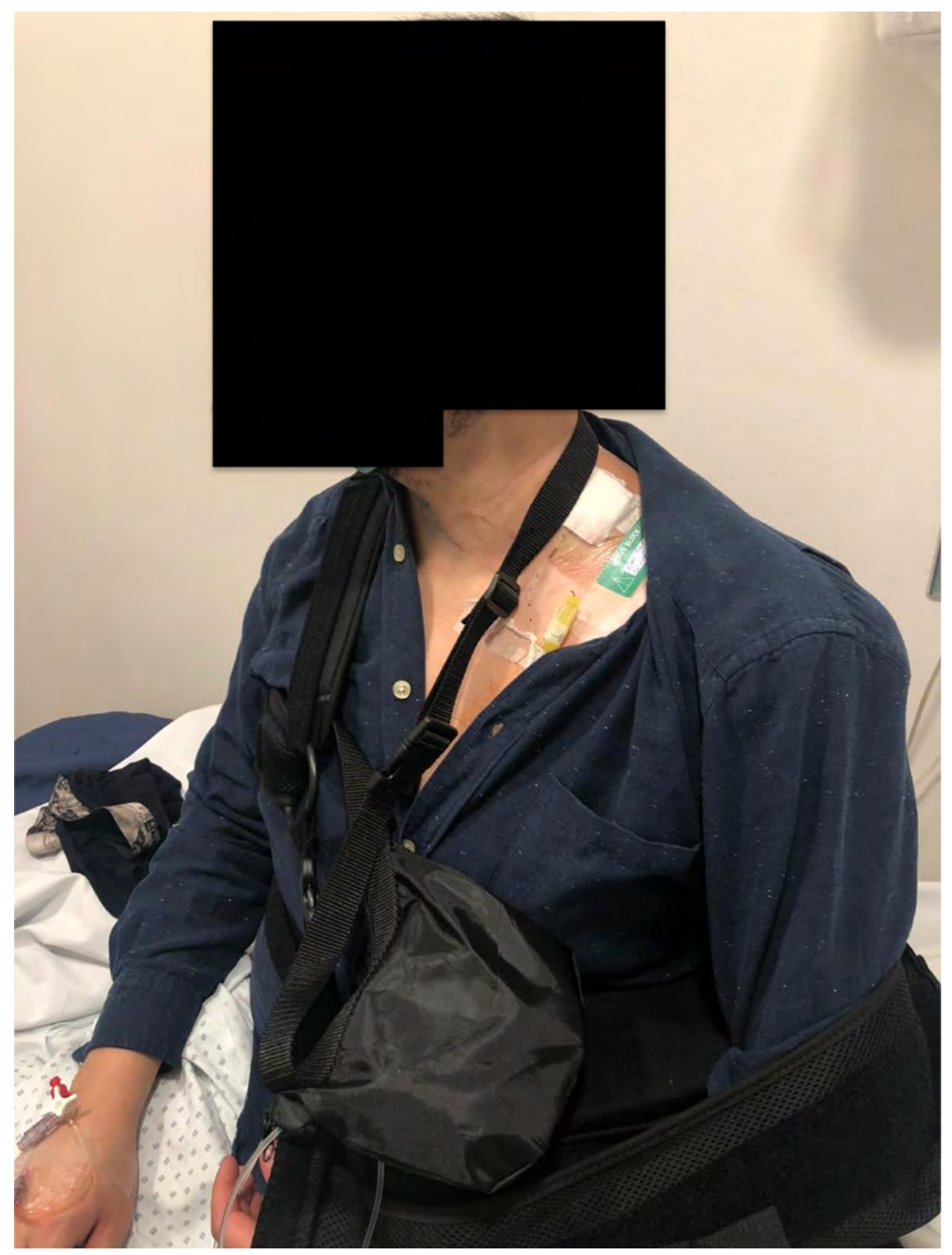

Foto 1. Bomba elastomérica con bolso colgante alrededor del cuello del paciente. 


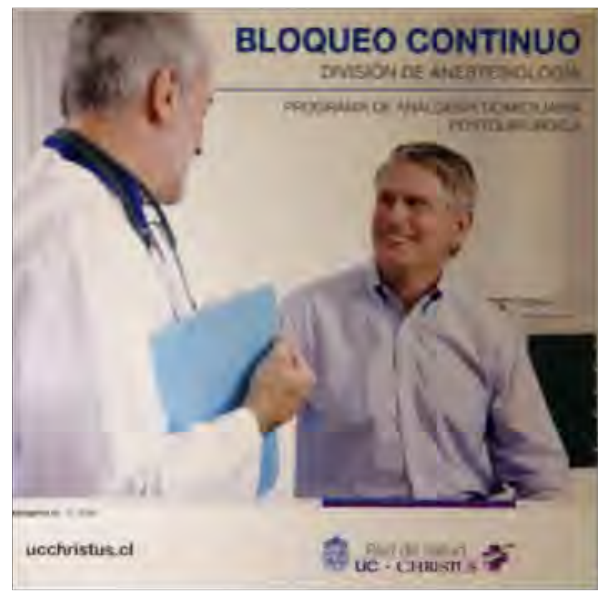

¿DÓNDE Y CUÁNDO CONSULTAR?

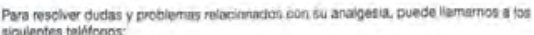

Lunes a viernos:

- De of:09 a 1700 horis, 22226398766 a 223543270.

Fines de semana y testivos:

- Comuniquese ai nintero coliue (56-9) 42116590

Si prosenta:

- Adormecimiento de labios o gusto metilico en la boca, dosconecte inmediatamente is

intusión y contáctese con nosteros. cuche pto5o zumbidos en

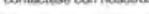

Comuniquese con nosotros sil presenta:

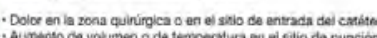

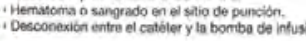

ucchristus.cl

Q6) Red de Salud tor

Nombre Paciente:

C.l:

cruois:

Tipo de noquas:

Fecha instalación cattiter:

Focha instalación bombus

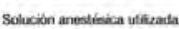

Fecha Ata:

Fochla que dibbe retirar of catieter:

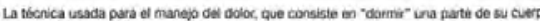

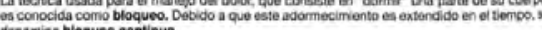
denarina bloqueo continuo.

Paral logite ef bloqueo contiouo se le ha instalado un catteter (un delgado tubo de plasticen)

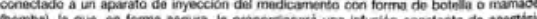

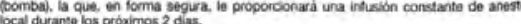

A mismo tiempo, usted continuara con los stouernies analgésicos orales:

1. Parcetamol. 1 gramo cada 8 horas $y / 0$ por 5 a 7 dias

3.

\section{Seguimiento:}

Usted sori controlado teleténicamente 1 vez at dia por es folf

Drial.

o par ofro integrante de la Unicac de Manejo des Dolor

Retrar of sistema:

Usted debe retirar su catiter el dia -a ias. - horas.

Es probable que la bornba estó vacia o quede muy poca cantidad de medcamento. debe seguir lass sovientes instinociones:

1. Rotive las tolis adhesivas que fijan el catiter a su piel.

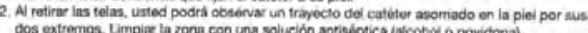

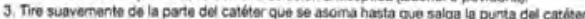

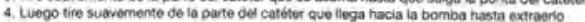

5. Uno vez finalizado el retiro del catíter, Unpie el sitio de punción con una solución

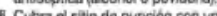

7. Trom a is basura eic catiene y la bamba de intusión. Recuerde que son desectrables $y$ no genera hesgo para usted o su entorno.

5) nocesita de asistencia para retira es sistema, puede dingmen a la Division de Arestusiologih

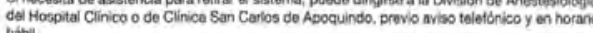

Foto 2. Díptico con la información que se les entrega a los pacientes de ARDC. 
CARSACH

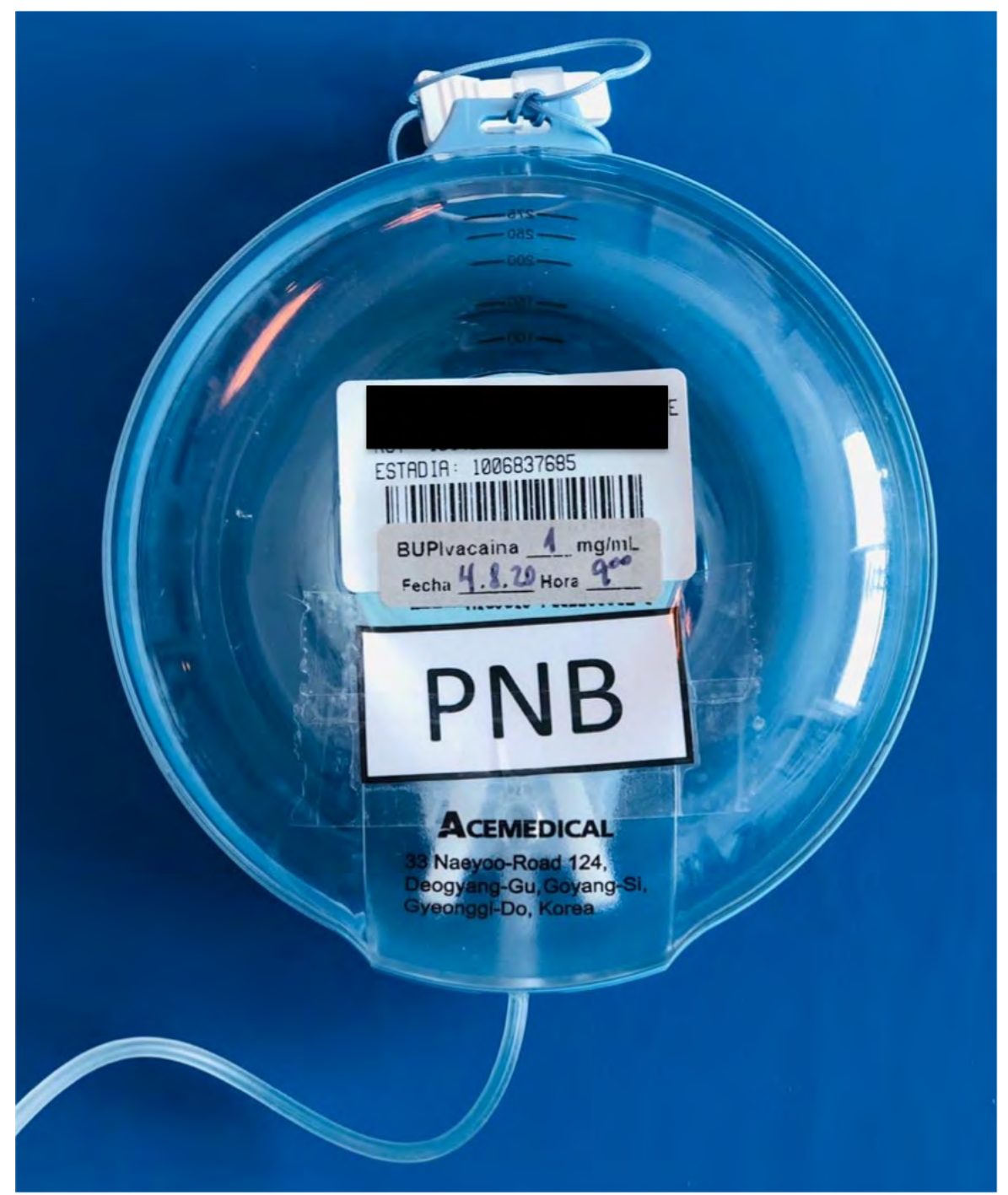

Foto 3 Bomba elastomérica rotulada con nombre del paciente, Rut, medicamento, fecha y hora de instalación. 


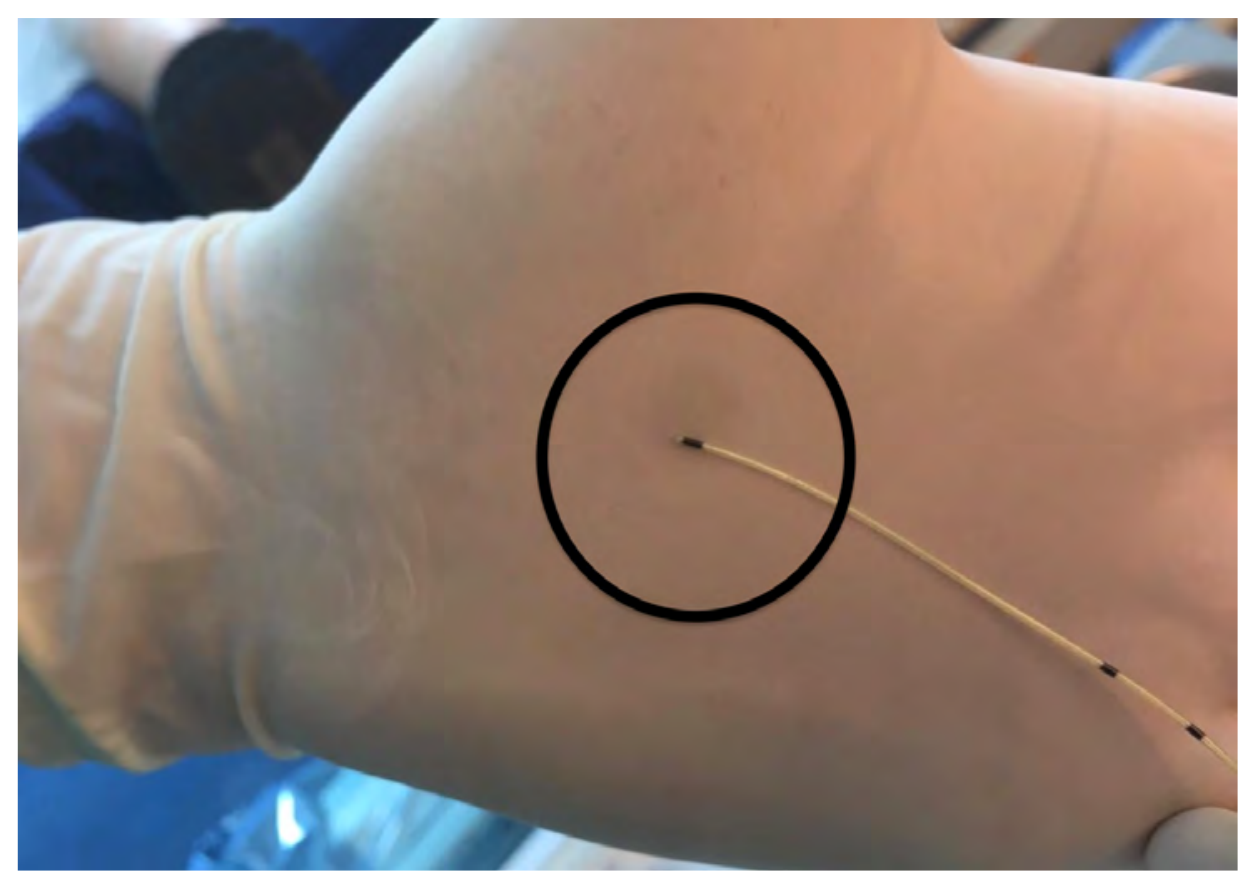

Foto 4. El extremo del catéter de nervio periférico es de otro color, eso es lo que se debe apreciar al momento de retirar el catéter. 
CARSACH

página 108 